\section{Moderated poster session 1}

\section{ASSESSMENT OF MARKERS OF CARDIAC TOXICITY FOLLOWING COMBINED TREATMENT OF CARDIOMYOCYTES WITH EPIRUBICIN AND TRASTUZUMA}

${ }^{1} \mathrm{C}$ Tonry, ${ }^{1} \mathrm{E}$ Mhic Donncha, ${ }^{1} \mathrm{H}$ Scott, ${ }^{2} \mathrm{M}$ Harbison, D Bell, ${ }^{1} \mathrm{C}$ Watson. ${ }^{1}$ Queens University Hospital Belfast, UK; ${ }^{2}$ Belfast Trust, UK

\subsection{6/heartjn|-2020-ICS.22}

Background Advancements in cancer therapy have significantly improved long-term survival rates of those with breast cancer. The addition of epirubicin treatment prior to standard trastuzumab treatment has been shown to slow progression of disease, reduce mortality and extend duration of survival in patients with HER2+ Breast Cancer. However, both drugs have off target cardiotoxic effects. Currently echocardiography is the only standardised diagnostic measure for detecting cardiac dysfunction. However, this is often after significant irreversible cardiac damage has occurred. There is an emerging need for blood-based biomarkers to aid in diagnosing subclinical cardiac dysfunction and to stratify those at risk prior to therapy.

Methods AC16 human cardiomyocytes were treated with Epirubicin $(2.6 \mathrm{ug} / \mathrm{ml})$ and Trastuzumab (150 ug/ml), both together and in monotherapy, over $10 \mathrm{hr}$ and $26 \mathrm{hr}$ timepoints. Cell viability was assessed via MTT cell viability assay. Protein and gene expression of Troponin I and BNP were assessed via western blot analysis and RT-PCR. Western blot analysis and fluorescent microscopy staining of oxidative stress markers was also carried out to elude to the potential mechanisms of cardiac damage.

Results Morphological changes occurred in all cells treated over 26 hrs, particularly with combined treatment. Cardiomyocytes treated with epirubicin alone showed the most significant reduction in cell viability compared to control ( $\mathrm{p} \leq$ $0.01 \%)$. Some increase in BNP and Troponin I expression was observed in cardiomyocytes treated with both epirubicin and trastuzumab. Pre-treatment of cardiomyocytes with recombinant BNP ameliorated chemotherapy-induced cell death in cardiomyocytes to some degree, however the effect was not significant.

Conclusion/Implications Combined treatment with epirubicin and trastuzumab exacerbates chemotherapy-induced cardiotoxicity. Troponin I and BNP are biomarkers that could be used as a diagnostic tool for prediction of subclinical chemotherapy-induced cardiotoxicty but further work is required to establish their true clinical utility.

\section{THE FIRST DESCRIPTION OF A SMARTPHONE-BASED EVALUATION OF THE CONJUNCTIVAL MICROCIRCULATION IN PATIENTS PRESENTING WITH ACUTE MYOCARDIAL INFARCTION}

${ }^{1} \mathrm{PF}$ Brennan, ${ }^{2} \mathrm{~A}$ Awuah, ${ }^{3} \mathrm{M}$ Jing, ${ }^{2} \mathrm{~A}$ McNeil, ${ }^{3} \mathrm{D}$ Finlay, ${ }^{2} \mathrm{~J}$ Mclaughlin, ${ }^{2} \mathrm{MA}$ Nesbit, ${ }^{4} \mathrm{E}$ Trucco, ${ }^{2} \mathrm{~T}$ Moore, ${ }^{1} \mathrm{MS}$ Spence. ${ }^{1}$ Royal Victoria Hospital, Belfast, UK; ${ }^{2}$ Ulster University, UK; ${ }^{3}$ NIBEC; ${ }^{4}$ VAMPIRE, University of Dundee, UK

10.1136/heartjnl-2020-ICS.23

Background Microcirculatory dysfunction and microvascular dysfunction occur early in the development of cardiovascular disease (CVD) with acute myocardial infarction (MI) being a late consequence of CVD. The conjunctival microcirculation is readily-accessible for quantitative assessment using a slit-lamp biomicroscope. We have previously reported the study of the conjunctival microcirculation in healthy volunteers and in patients with cyanotic congenital heart disease.

Methods We performed conjunctival microcirculatory assessment in a group of inpatients with acute type $1 \mathrm{MI}$ and in age/sex-matched healthy controls. Image acquisition and video capture was performed using an iPhone 6s combined with a slit-lamp biomicroscope. The conjunctival vessels in each hemisphere (temporal/nasal) of both eyes were studied. Microcirculatory parameters quantified included axial velocity, wall shear rate and blood volume flow.

Results Conjunctival microcirculatory assessment was assessed

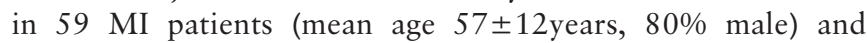

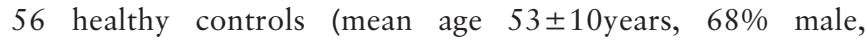
mean QRISK-3 score 8.1 $\pm 7.6 \%)$. STEMI and NSTEMI made up $36 \%(n=21)$ and $64 \%(n=38)$ of the MI patient group, respectively. Baseline characteristics are summarised in table 1.

A total of 4163 vessel segments (healthy control 1904 total, 34 per patient vs. MI 2259 total, 38 per patient) were analysed for the two groups. Mean conjunctival microvessel diameter was $21.41 \pm 7.57$ um for the controls which was significantly lower than the 22.32 \pm 7.66 um seen in MI patients $(p<0.0005)$. Axial velocity for the MI patients was significantly lower at $0.49 \pm 0.17 \mathrm{~mm} / \mathrm{s}$ compared to $0.53 \pm 0.15 \mathrm{~mm} / \mathrm{s}$ for the controls $(\mathrm{p}<0.0005$ (figure 1)). Wall shear rate was also significantly lower in the MI group (144.96 $\pm 88.45 \mathrm{~s}-1$ vs. $162 \pm 93 \mathrm{~s}-1, \mathrm{p}<0.0005$ (figure 2)). There was no significant difference in blood volume flow between the MI and controls $(154 \pm 124.8 \mathrm{pl} / \mathrm{s}$ vs. $152.6 \pm 124.4 \mathrm{pl} / \mathrm{s}, \mathrm{p} 0.84)$.

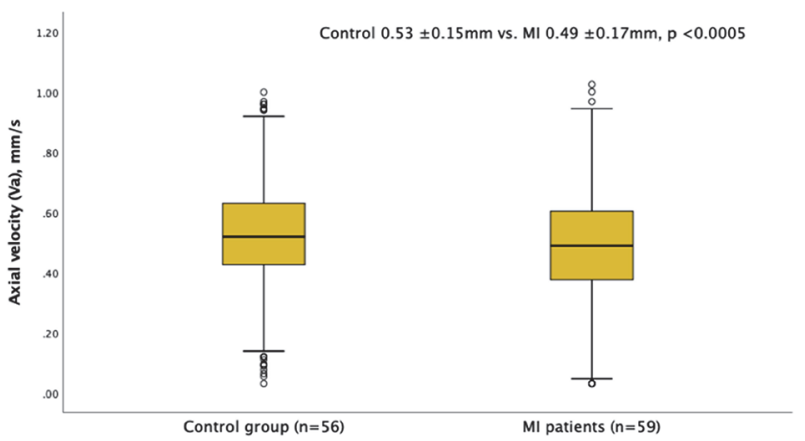

Abstract 23 Figure 1 Axial velocity

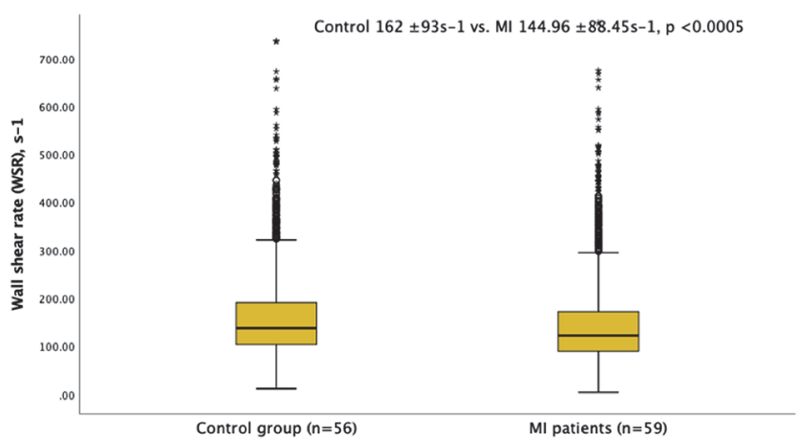

Abstract 23 Figure 2 Wall shear rate 


\begin{tabular}{llll} 
Abstract 23 Table 1 & Baseline characteristics & \\
\hline Baseline characteristic & $\begin{array}{l}\text { Control } \\
(\mathbf{n}=56)\end{array}$ & MI & p value \\
& $(\mathbf{n}=59)$ & \\
\hline Age, years \pm SD & $53 \pm 10$ & $57 \pm 12$ & 0.07 \\
Male sex & $38(68)$ & $47(80)$ & 0.15 \\
Q-Risk 3\% & $8.1 \pm 7.6$ & $14.3 \pm 9.4$ & $<0.0005$ \\
IHD & 0 & $11(18.6)$ & $<0.0005$ \\
Prior MI & 0 & $8(13.6)$ & 0.006 \\
Prior stroke & 0 & $1(1.7)$ & 1 \\
Hypertension & $7(12.5)$ & $21(35.6)$ & 0.004 \\
Diabetes mellitus & 0 & $12(20.3)$ & 0.002 \\
Dyslipidaemia & $11(19.6)$ & $25(42.4)$ & 0.009 \\
Smoking history & $21(37.5)$ & $36(61)$ & 0.01 \\
COPD & $4(7.1)$ & $2(3.4)$ & 0.43 \\
Creatine clearance, ml/ & $84 \pm 29$ & $91 \pm 37$ & 0.56 \\
min & & & \\
Haemoglobin, g/l & $146 \pm 11$ & $142 \pm 15$ & 0.08 \\
Haematocrit, l/l & $0.43 \pm .03$ & $0.42 \pm .04$ & 0.29 \\
Platelet count, 10 & $269 \pm 43 \pm 64$ & 0.27 \\
\hline
\end{tabular}

Conclusions Using our novel imaging system, alterations in conjunctival microcirculatory parameters for MI patients compared to healthy controls were found. Axial velocity and wall shear rate were significantly lower in the MI group, similar to what we previously reported in patients with cyanotic congenital heart disease. These alterations in conjunctival microcirculatory parameters are suggestive of endothelial dysfunction and application of this system may enhance future assessment of CVD risk.

\section{\begin{tabular}{|l|l}
\hline 24 & SGLT-2I THERAPY IN HEART FAILURE : CHALLENGES
\end{tabular} AND OPPORTUNITIES}

A Radhakrishna, R Cusack, J Barton. University Hospital Galway, Galway, Ireland

\subsection{6/heartjnl-2020-ICS.24}

Introduction Heart failure (HF) is a complex disease which is growing to be a significant cause of morbidity and mortality leading to increased cost of chronic care and hospitalization. In the DAPA-HF study, the sodium-glucose co-transporter 2 inhibitor (SGLT-2i) dapagliflozin was shown to reduce the risk of worsening $\mathrm{HF}$ and death in patients with $\mathrm{HF}$ with reduced ejection fraction (HFrEF). Our goal was to conduct an audit in a tertiary referral centre at University Hospital Galway (UHG) to identify patients with HFrEF who fulfil the eligibility criteria for SGLT-2i therapy, as seen in the DAPA-HF study. We also sought to identify patients with Type 2 Diabetes Mellitus (T2DM) in our HFrEF cohort who are potential candidates for improvement of glycaemic control with SGLT-2i therapy according to the ADA-EASD Guidelines.

Methodology A retrospective audit was conducted on 129 patients with HFrEF attending the specialist-led heart failure clinic at UHG between January and March 2020. Demographic, clinical, biochemical and medication data were collected from medical charts and our local digital database: EVOLVE $^{\circledR}$ and $\mathrm{CVWeb}^{\circledR}$. Patients had to meet the DAPA-HF inclusion criteria to be deemed eligible for dapagliflozin therapy.
Results Table 1 summarises the baseline clinical data and table 2 summarises the list of medical therapy at our centre. Of note, the 129 patients in our study represented a more elderly cohort compared to the DAPA-HF study population.

Only 49/129 (38\%) of our HFrEF patients were eligible for SGLT-2i therapy based on the DAPA-HF inclusion criteria. This is primarily due to the higher than expected percentage of patients in our cohort who were asymptomatic (34.9\%) and who had low NT-proBNP levels (29.6\%). 16/ $129(12.4 \%)$ had severe CKD with an eGFR $<30 \mathrm{ml} / \mathrm{min} /$ $1.73 \mathrm{~m}^{2}$.

There were only 26/129 (20.2\%) patients with T2DM of which 6 patients were already on SGLT-2i. The majority had ischemic cardiomyopathy (69\%) with concomitant risk factors and $(30.8 \%)$ had poor glycaemic control.

Conclusion This study shows a lower than expected number of patients in our centre who would have been included in the DAPA-HF trial. This could be because many patients in

Abstract 24 Table 1 Baseline characteristics and co-morbidities

\begin{tabular}{lll}
\hline & Local Data ( $\mathbf{n}=129)$ & DAPA-HF ( $\mathbf{n}=4744)$ \\
\hline Mean Age (Years) & $71.3 \pm(11.8)$ & 66 \\
Male (\%) & 69 & 77 \\
T2DM (\%) & 20.2 & 45 \\
NYHA Class (\%) & & \\
NYHA I & 34.9 & None \\
NYHA II & 48.1 & 68 \\
NYHA III & 15.5 & 32 \\
NYHA IV & 1.5 & 1 \\
Mean Left Ventricular & $28.5 \pm(7.4)$ & 31 \\
Ejection Fraction (\%) & & \\
Mean Systolic BP (mmHg) & $122.7 \pm(17)$ & 122 \\
Median NT-proBNP (pg/mL) & 1317 & 1437 \\
Mean eGFR (ml/min/1.73 m²) & $57.4 \pm(20.5)$ & 66 \\
Ischemic Aetiology (\%) & 58.1 & 56 \\
HF Hospitalization within 1 year (\%) & 36.4 & 47 \\
Hypertension (\%) & 77.5 & 74 \\
Atrial Fibrillation/Flutter\% & 48 & 40 \\
\hline
\end{tabular}

Abstract 24 Table 2 Treatment

\begin{tabular}{lll}
\hline & Local Data $(\mathrm{n}=129)$ & DAPA-HF $(\mathrm{n}=\mathbf{4 7 4 4})$ \\
\hline Diuretic (\%) & 67.4 & 93 \\
ACEI (\%) & 36.4 & 56 \\
ARB (\%) & 8.5 & 28 \\
ACEI/ARB/ARNI (\%) & 90.7 & 93.5 \\
Beta-Blocker (\%) & 93.8 & 96 \\
MRA (\%) & 27.9 & 71 \\
ICD (\%) & 20.2 & 26 \\
CRT (\%) & 10.9 & 7 \\
Diabetes Medication $(\mathbf{n}=26)$ & & \\
Metformin & 10 & \\
Sulphonylurea & 3 & \\
DPP4 Inhibitor & 10 & \\
GLP-1 Agonist & 0 & \\
Insulin & 3 & \\
SGLT-2i & 6 & \\
\hline
\end{tabular}

